\title{
BMJ
}

\section{Antibiotic treatment for pyelonephritis in children: multicentre randomised controlled non-inferiority trial}

\author{
Giovanni Montini, consultant in paediatric nephrology,, Antonella Toffolo, consultant in paediatrics, ${ }^{1}$ \\ Pietro Zucchetta, consultant in nuclear medicine, ${ }^{3}$ Roberto Dall'Amico, consultant in paediatrics, ${ }^{4}$ \\ Daniela Gobber, senior lecturer in epidemiology, ${ }^{5}$ Alessandro Calderan, general practitioner, ${ }^{6}$ \\ Francesca Maschio, consultant in paediatrics, ${ }^{7}$ Luigi Pavanello, consultant in paediatrics, ${ }^{8}$ Pier Paolo \\ Molinari, consultant in paediatrics, ${ }^{9}$ Dante Scorrano, consultant in paediatrics, ${ }^{10}$ \\ Sergio Zanchetta, consultant in paediatrics, ${ }^{11}$ Walburga Cassar, consultant in paediatrics, ${ }^{12}$ \\ Paolo Brisotto, consultant in paediatrics, ${ }^{13}$ Andrea Corsini, consultant in paediatrics, ${ }^{14}$ Stefano Sartori, fellow \\ in paediatrics, ${ }^{1}$ Liviana Da Dalt, associate professor, paediatric emergency, ${ }^{15}$ Luisa Murer, consultant in \\ paediatric nephrology, ${ }^{1}$ Graziella Zacchello associate professor, paediatric nephrology ${ }^{1}$
}

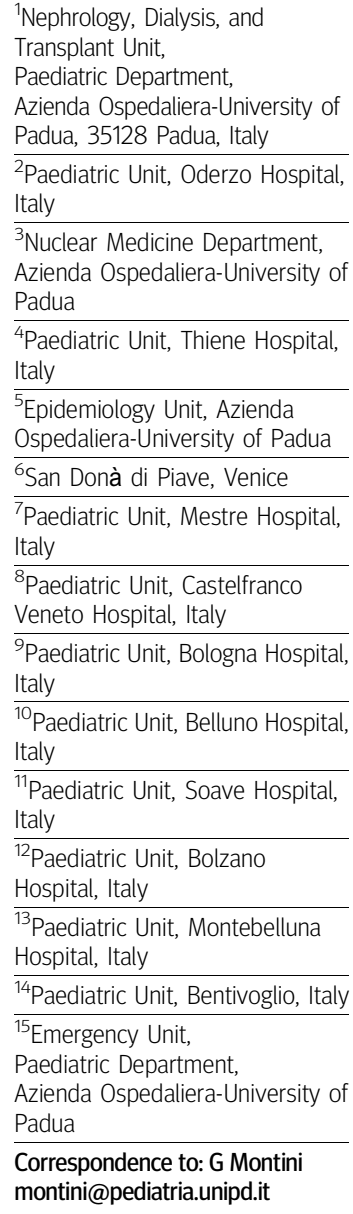

${ }^{4}$ Paediatric Unit, Thiene Hospital, Italy

${ }^{5}$ Epidemiology Unit, Azienda Ospedaliera-University of Padua

${ }^{6}$ San Donà di Piave, Venice

${ }^{7}$ Paediatric Unit, Mestre Hospital, Italy

${ }^{8}$ Paediatric Unit, Castelfranco Veneto Hospital, Italy

${ }^{9}$ Paediatric Unit, Bologna Hospital, Italy

${ }^{10}$ Paediatric Unit, Belluno Hospital, Italy

${ }^{11}$ Paediatric Unit, Soave Hospital, Italy

${ }^{12}$ Paediatric Unit, Bolzano

Hospital, Italy

${ }^{13}$ Paediatric Unit, Montebelluna Hospital, Italy

${ }^{14}$ Paediatric Unit, Bentivoglio, Italy

${ }^{15}$ Emergency Unit,

Paediatric Department.

Azienda Ospedaliera-University of Padua

Correspondence to: G Montini montini@pediatria.unipd.it

doi:10.1136/bmj.39244.692442.55

\section{ABSTRACT}

Objective To compare the efficacy of oral antibiotic treatment alone with treatment started parenterally and completed orally in children with a first episode of acute pyelonephritis.

Design Multicentre, randomised controlled, open labelled, parallel group, non-inferiority trial.

Setting 28 paediatric units in north east Italy.

Participants 502 children aged 1 month to $<7$ years with clinical pyelonephritis.

Intervention Oral co-amoxiclav $(50 \mathrm{mg} / \mathrm{kg} /$ day in three doses for 10 days) or parenteral ceftriaxone $(50 \mathrm{mg} / \mathrm{kg} /$ day in a single parenteral dose) for three days, followed by oral co-amoxiclav $(50 \mathrm{mg} / \mathrm{kg} /$ day in three divided doses for seven days).

Main outcomes measures Primary outcome was the rate of renal scarring. Secondary measures of efficacy were time to defervescence $\left(337^{\circ} \mathrm{C}\right)$, reduction in inflammatory indices, and percentage with sterile urine after 72 hours. An exploratory subgroup analysis was conducted in the children in whom pyelonephritis was confirmed by dimercaptosuccinic acid (DMSA) scintigraphy within 10 days after study entry.

Results Intention to treat analysis showed no significant differences between oral $(n=244)$ and parenteral $(n=258)$ treatment, both in the primary outcome (scarring scintigraphy at 12 months $27 / 197(13.7 \%) v 36 / 203$ $(17.7 \%)$, difference in risk $-4 \%, 95 \%$ confidence interval $-11.1 \%$ to $3.1 \%$ ) and secondary outcomes (time to defervescence 36.9 hours (SD 19.7) v 34.3 hours (SD 20), mean difference 2.6 ( -0.9 to 6.0 ); white cell count $9.8 \times 10^{9} / /(S D 3.5) \vee 9.5 \times 10^{9} / /$ (SD 3.1), mean difference 0.3 ( -0.3 to 0.9$)$; percentage with sterile urine $185 / 186 \mathrm{v}$ $203 / 204$, risk difference $-0.05 \%(-1.5 \%$ to $1.4 \%)$ ).

Similar results were found in the subgroup of 278 children with confirmed acute pyelonephritis on scintigraphy at study entry.
Conclusions Treatment with oral antibiotics is as effective as parenteral then oral treatment in the management of the first episode of clinical pyelonephritis in children. Trial registration Clinical Trials NCT00161330.

\section{INTRODUCTION}

Acute pyelonephritis is one of the most common serious bacterial infections in childhood, particularly in young children, ${ }^{1-3}$ because of the potential renal scarring. ${ }^{4-8}$ The diagnosis is based on the clinical presentation (clinical pyelonephritis), with fever and raised inflammatory indices, active urinary sediment, and subsequent positive results on urine culture. Published guidelines recommend initial treatment with a parenteral third generation cephalosporin followed by oral antibiotics..$^{9-11}$

A recent Cochrane review of antibiotic treatment for acute pyelonephritis in children identified 18 randomised trials that for the most part compared different antibiotic regimens given parenterally. ${ }^{11}$ There were no significant differences in the risk of persistent renal damage (three trials, 315 children: relative risk 0.99 , 95\% confidence interval 0.72 to 1.37 ) between initial intravenous (three to four days) followed by oral treatment and completely intravenous treatment (seven to 14 days). Only one previous randomised controlled trial (306 children) compared oral treatment (cefixime) only with antibiotics started parenterally. ${ }^{12}$ There was no significant difference between the two groups in terms of renal scarring at six months $(1.45,0.69$ to 3.03). Hoberman et al expressed some concern regarding the low rate of scarring in both groups in the study compared with the rates reported elsewhere. ${ }^{12}$ Furthermore, $90 \%$ of children studied were girls. This was despite the mean age of the children being 8 months, and studies have shown that the sex distribution in the first 6 months is about equal, with a significant predominance in girls only after 12 months of age. ${ }^{13}$ 
Children with presumed acute pyelonephritis (APN) $(n=502)$

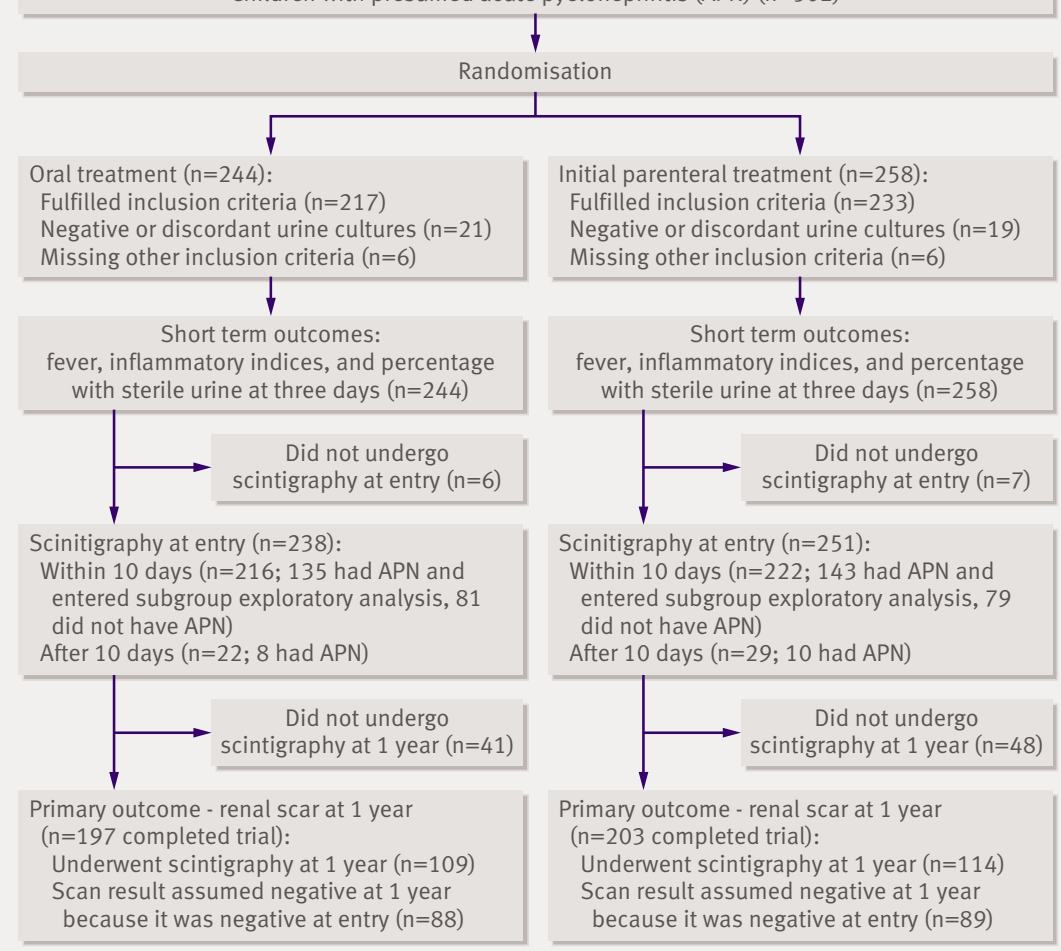

Fig 1 | Flow of patients through study and adherence to protocol

We conducted a non-inferiority randomised controlled trial to determine whether an entirely oral treatment with co-amoxiclav is therapeutically similar to an initial parenteral treatment with ceftriaxone followed by oral co-amoxiclav in children with clinical acute pyelonephritis. Oral treatment is easier to use and does not require admission to hospital, leading to reduced costs.

\section{METHODS}

The study was a randomised controlled, multicentre, open labelled, parallel group, non-inferiority trial. It was performed from June 2000 to July 2005 at 28 paediatric units located in north east Italy and was coordinated by the unit of nephrology, dialysis, and transplantation of the paediatric department of Padua. The parents of each child who participated gave written informed consent before the study.

\section{Diagnosis}

Recruited children were aged from 1 month to $<7$ years and had a clinical diagnosis of acute pyelonephritis at presentation according to urinalysis (two concordant consecutive test results with white cell counts $\geq 25 / \mu 1$, $=1+$ with dipstick) and urine culture (two concordant consecutive tests with growth of only one micro-organism $\geq 100000$ colony forming units/ml). Urine was collected in a sterile urine bag; the two concordant consecutive urinalyses and cultures were required to minimise the risk of false positive results. Children also had to have at least two of fever $\geq 38^{\circ} \mathrm{C}$; inflammatory indices in the first 48 hours (erythrocyte sedimentation rate $\geq 30 \mathrm{~mm}$ in the first hour or $C$ reactive protein $\geq 3$ times upper limit of normal values, or both); and neutrophil count above the normal values for age. ${ }^{14}$

All recruited children were admitted to hospital and remained there until their temperature was normal or for at least three days. The parenchymal localisation of the infection was confirmed by an acute positive result on dimercaptosuccinic acid (DMSA) scintigraphy in those children who underwent the procedure within 10 days after the start of antibiotic treatment.

\section{Inclusion and exclusion criteria}

All children had to have had normal findings on prenatal ultrasonography, no history of acute pyelonephritis. We excluded children with severe clinical sepsis, dehydration, and vomiting, which precluded administration of oral antibiotics; ongoing antibiotic treatment; allergy to the study drugs; creatinine clearance $\left(\right.$ Schwartz $^{15}$ formula) $\leq 70 \mathrm{ml} / \mathrm{min} / 1.73 \mathrm{~m}^{2}$.

The eligibility criteria are similar to those in the previous trials that established the efficacy of the initial parenteral treatment followed by oral antibiotic as reported in the Cochrane review. ${ }^{11}$

\section{Interventions}

After recruitment, children were allocated to oral treatment with co-amoxiclav $50 \mathrm{mg} / \mathrm{kg} /$ day in three doses for 10 days (new treatment) or initial parenteral treatment with ceftriaxone $50 \mathrm{mg} / \mathrm{kg}$ /day in a single dose for three days, followed by oral co-amoxiclav $50 \mathrm{mg}$ / $\mathrm{kg} /$ day in three doses for seven days (standard treatment). The standard treatment is similar to the one used in the trials that established its efficacy ${ }^{11}$ (intravenous antibiotic for three to four days followed by oral antibiotic treatment for a total duration of 10 to 21 days).

We modified treatment in children whose health deteriorated in the 48 hours after the start of treatment; those with persistent fever $\left(>38^{\circ} \mathrm{C}\right)$ in the 72 hours after the start of treatment; and those with intolerance or an adverse reaction to the drugs used. The children's axillary temperature was measured every six hours. Inflammatory indices, urinalyses, and urine culture were repeated on the third day of treatment.

\section{Imaging studies}

Ultrasonography and scintigraphy were planned no later than 10 days after the start of antibiotic treatment. After treatment was completed, children remained on antibiotic prophylaxis until voiding cystography (radiology, with one filling) was carried out (within two months). If children had a positive result on scintigraphy for acute pyelonephritis we scheduled a repeat scan after one year to detect any renal scarring at the site of the original pyelonephritis. In children with a negative result we considered it unethical to carry out a repeat scan. As previous studies have shown that all the children whose initial scan result was normal had 
normal scans at follow-up, ${ }^{12}$ we considered negative results at entry to mean negative results at 12 months for the analyses. We performed static renal scintigraphy three to four hours after injection of a weight scaled dose of technetium-99m DMSA to obtain views in the posterior and both posterior oblique projections for 300 kilocounts or more. Focal or diffuse areas of decreased uptake in the first scan, without evidence of cortical loss, indicated acute pyelonephritis. Renal scarring was defined as decreased uptake with distortion of the contours or as cortical thinning with loss of parenchymal volume. Two nuclear physicians, blinded to the test results, interpreted the scans independently and resolved discrepancies by discussion.

\section{Outcome measurements}

Our primary end point was the rate of renal scarring after 12 months. The secondary outcome was the

$\overline{\text { Table } 1 \text { Demographic and clinical characteristics of } 502 \text { children with acute pyelonephritis (APN) }}$ according to allocation to new treatment (oral co-amoxiclav) or standard treatment (intravenous ceftriaxone followed by oral co-amoxiclav)

New treatment $(n=244)$

Standard treatment $(n=258)$

Age (months):

\begin{tabular}{|c|c|c|}
\hline Median (range) & $8.1(1-81)$ & $7.9(1-99)$ \\
\hline Mean (SD) & $12.7(14.2)$ & $11.9(13.9)$ \\
\hline No of children & 244 & 258 \\
\hline No (\%) girls & $159 / 244(65.2)$ & $163 / 258(63.2)$ \\
\hline \multicolumn{3}{|l|}{ Max body temperature $\left({ }^{\circ} \mathrm{C}\right)$ : } \\
\hline Median (range) & $39.25(36.5-41)$ & $39.2(36.8-41.5)$ \\
\hline Mean (SD) & $39.1(0.77)$ & $39.2(0.78)$ \\
\hline No of children & 240 & 255 \\
\hline \multicolumn{3}{|l|}{ White cell count $\left(\times 10^{9} / \mathrm{l}\right)$ : } \\
\hline Median (range) & $17.2(5.5-45.9)$ & $17.0(3.8-37.1)$ \\
\hline Mean (SD) & $18.1(6.4)$ & $17.8(6.0)$ \\
\hline No of children & 243 & 257 \\
\hline \multicolumn{3}{|l|}{ Neutrophils $\left(\times 10^{9} / \mathrm{l}\right)$ : } \\
\hline Median (range) & $9.6(1.5-29.1)$ & $9.9(1.6-29.1)$ \\
\hline Mean (SD) & $10.8(5.0)$ & $10.7(5.0)$ \\
\hline No of children & 218 & 210 \\
\hline \multicolumn{3}{|c|}{ Erythrocyte sedimentation rate ( $\mathrm{mm}$ in first hour): } \\
\hline Median (range) & $58(1-150)$ & $65(1-122)$ \\
\hline Mean (SD) & $59.7(28.9)$ & $66(28.5)$ \\
\hline No of children & 153 & 150 \\
\hline \multicolumn{3}{|l|}{$C$ reactive protein $(\mathrm{mg} / \mathrm{l})^{\star}$ : } \\
\hline Median (range) & $12.5(0.1-168)$ & $14.2(0.1-172)$ \\
\hline Mean (SD) & $17.9(19.6)$ & $19.6(20.6)$ \\
\hline No of children & 243 & 257 \\
\hline No (\%) with confirmed APN† & $135 / 216(62.5)$ & $143 / 222(64.4)$ \\
\hline No (\%) with VUR & $53 / 231(22.9)$ & $49 / 242(20.2)$ \\
\hline
\end{tabular}

VUR grade:

\begin{tabular}{lcc}
\hline 1 & 11 & 13 \\
\hline 2 & 18 & 19 \\
\hline 3 & 21 & 13 \\
\hline 4 & 3 & 3 \\
\hline 5 & 0 & 1 \\
\hline
\end{tabular}

VUR=vesicoureteric reflux.

*Ratio between obtained value and upper limit of normal reference values for each laboratory.

tConfirmed with scintigraphy in children who underwent scan within 10 days after start of antibiotic treatment. efficacy of antibiotic treatment in the short term: time to defervescence (axillary temperature $<37^{\circ} \mathrm{C}$ ), reduction in inflammatory indices, and percentage with sterile urine 72 hours after the start of treatment.

We carried out an exploratory subgroup analysis of primary and secondary outcomes in the group of children with pyelonephritis confirmed by scintigraphy performed within 10 days after the start of antibiotic treatment. Our primary outcome is similar to the one that established the efficacy of initial intravenous therapy (three to four days) followed by oral antibiotic in three trials (315 children) recently reported by a Cochrane review. ${ }^{11}$

We compared the safety and acceptability of treatment in terms of the rate of discontinuation of treatment and the incidence of side effects.

\section{Randomisation}

The coordinating centre computer generated the randomisation scheme. Randomisation was stratified for sex and age ( $<2$ years $v \geq 2$ years). Each participating centre received four series (one for each stratum) of 10 allocation codes (five for oral co-amoxiclav and five for parenteral ceftriaxone) in sealed and sequentially numbered opaque envelopes. The sequence was concealed until interventions were assigned. Each participating centre allocated the children following the numeric order. The coordinating centre provided further envelopes as necessary.

We organised a series of meetings for investigators to standardise good clinical practice guidelines before the start of the trial and during the course of the study. The correctness of the randomisation was evaluated for each centre at the end of enrolment by comparing the patients' allocations with the copy of the randomisation list stored by the coordinating centre. We could not blind group assignment because of the different routes of administration of the drug.

\section{Statistical analysis}

Calculation of sample size-In around 15\% of children with acute pyelonephritis treated with parenteral antibiotics, scintigraphy 12 months after infection shows measureable renal scars. ${ }^{1-12}$ We therefore considered that an upper confidence limit of $25 \%$ for the new (oral) treatment would indicate non-inferiority. As previously suggested ${ }^{16}$ we adopted $10 \%$ as the margin of equivalence. We therefore required 220 children per group, with $90 \%$ power and $5 \% \alpha$ error for a one tailed test.

Analysis plan - We analysed data on all the children initially included in the trial according to the intention to treat principle. We used $\chi^{2}$ tests (Pearson's or Fisher's test) for binomial outcome measures and Student's $t$ test or the Mann-Whitney test for continuous outcome measures, as appropriate. We rejected the null hypothesis for all tests with two tailed $\alpha<0.05$. We calculated the differences between treatments with $95 \%$ confidence intervals and analysed skewed distributions on $\log$ scale with results transformed on linear scales. Stata 8.0 was used for the statistical analysis. 
RESULTS

Patients

The paediatric departments participating in the trial recruited 502 children from June 2000 to June 2004 among those seen because of fever or other symptoms indicating pyelonephritis. Follow-up was completed in July 2005. Figure 1 shows the flow of children through the trial and adherence to protocol. Immediately after recruitment and informed consent, children were randomised to either oral antibiotic (new treatment, $\mathrm{n}=244$ ) or parenteral antibiotic (standard treatment, $\mathrm{n}=258$ ). All patients enrolled were correctly allocated to their randomisation group. We analysed data from all the 502 randomised children (intention to treat analysis), including 40 (21 in the new treatment group and 19 in the standard treatment group) with negative or discordant urine cultures and 12 (six from each group) wrongly enrolled for other inclusion criteria (two were out of the age range, 10 did not fulfil the diagnostic criteria for pyelonephritis). We had data on the short term outcomes for all the 502 randomised children. Four hundred children completed the trial and were measured for renal scarring-the primary outcome (223 underwent scintigraphy at 12 months; 177 were considered to have negative results at 12 months because they had negative results at study entry). After they completed antibiotic treatment, 102 $(20.3 \%)$ patients were lost to follow up; with no significant difference between the groups. Of the 223 patients who underwent the renal scan at 12 months, scintigraphy at entry had confirmed pyelonephritis in 207 and was negative in 16 . These 16 were scanned at 12 months for reasons other than the protocol and, as expected, the scan result was negative.

There was no significant difference in adherence to protocol for scintigraphy at entry in the two groups. Thirteen children did not undergo a scan at study entry and 51 did not do so within 10 days. Of the 438 patients who underwent scintigraphy within 10 days, in 278 (135/216 in the new treatment group and 143/ 222 in standard treatment group) the results confirmed acute pyelonephritis, and these children constituted the population for the subgroup analysis. Those with

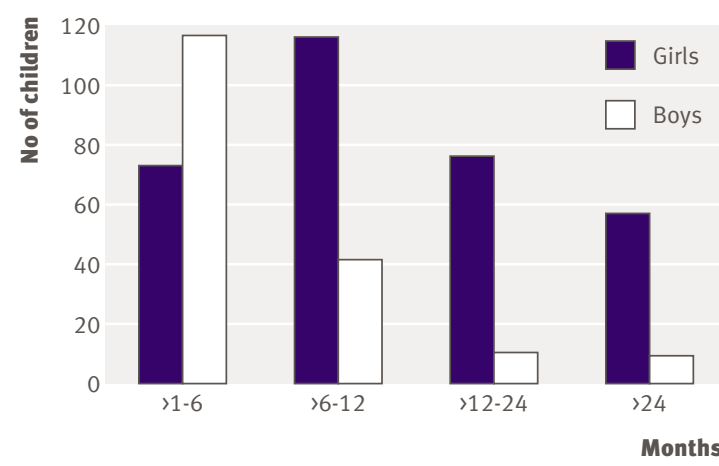

Fig 2 | Distribution by age (months) and sex of 502 children

a negative result at entry were considered to have a febrile urinary tract infection (UTI) in the absence of acute pyelonephritis.

Table 1 and figure 2 show the demographic and baseline data. There were no significant differences between the two groups.

Escherichia coli was the pathogen responsible in $94.4 \%(436 / 462)$ of confirmed urine cultures that showed bacteria growth. Bacterial resistance to protocol antibiotics was $6 \%(25 / 407)$ with co-amoxiclav and $<1 \%(3 / 343)$ with ceftriaxone.

\section{Primary outcome}

Scarring on scintigraphy at 12 months was not significantly different between the two groups: $27 / 197$ $(13.7 \%)$ in the new treatment group $v 36 / 203(17.7 \%)$ in the standard treatment group; risk difference $-4 \%$, $95 \%$ confidence interval $-11.1 \%$ to $3.1 \%$ (table 2 ). The exploratory subgroup analysis confirmed that the oral treatment was not inferior: 26/96 (27.8\%) v 33/100 $(33.0 \%)$; risk difference $-5.8 \%,-18.7 \%$ to $6.9 \%$ (table 3).

We found similar results when we counted all patients lost to follow-up in both groups as having a scar at 12 months (risk difference $-4.9 \%,-13.1 \%$ to $3.3 \%)$.

Table 2 | Primary and secondary outcomes according to treatment in the 502 randomised children according to allocation to new treatment (oral co-amoxiclav) or standard treatment (intravenous ceftriaxone followed by oral co-amoxiclav). Figures are means (SD) unless specified otherwise

\begin{tabular}{|c|c|c|c|}
\hline Parameter & New treatment $(n=244)$ & Standard treatment $(n=258)$ & Mean difference $(95 \% \mathrm{Cl})$ \\
\hline \multicolumn{4}{|l|}{ Short term outcomes } \\
\hline Time to defervescence (hours) & $36.9(19.7)(n=241)$ & $34.3(20)(n=253)$ & $2.6(-0.9$ to 6$)$ \\
\hline White cell count $\left(\times 10^{9} / 1\right)^{*}$ & $9.8(3.5)(n=230)$ & $9.5(3.1)(n=243)$ & $0.3(-0.3$ to 0.9$)$ \\
\hline Neutrophils $\left(\times 10^{9} / \mathrm{l}\right)^{\star}$ & $3.0(2.2)(n=207)$ & $2.8(1.9)(n=217)$ & $0.2(-0.2$ to 0.6$)$ \\
\hline $\begin{array}{l}\text { Erythrocyte sedimentation rate ( } \mathrm{mm} \text { in first } \\
\text { hour)* }\end{array}$ & $50.8(32)(n=170)$ & $52.6(27.9)(n=168)$ & $-1.8(-8.2$ to 4.7$)$ \\
\hline$C$ reactive protein $(\mathrm{mg} / \mathrm{l})^{*} \dagger$ & $9.3(20.9)(n=235)$ & $8.2(15.4)(n=251)$ & $1.1(-2.6$ to 4.1$)$ \\
\hline Sterile urine & $185 / 186(99.45 \%)$ & $203 / 204$ (99.5\%) & $-0.05 \%(-1.5 \%$ to $1.4 \%)$ \\
\hline \multicolumn{4}{|l|}{ Primary outcome } \\
\hline Scar on renal scan at 12 months & $27 / 197(13.7 \%)$ & $36 / 203(17.7 \%)$ & $-4 \%(-11.1 \%$ to $3.1 \%)$ \\
\hline
\end{tabular}

*Parameters obtained 72 hours after start of antibiotic treatment.

†Ratio between obtained value and upper limit of normal reference values for each laboratory. 
Table 3 | Primary and secondary outcomes according to treatment in 278 children with confirmed APN on DMSA at entry according to allocation to new treatment (oral co-amoxiclav) or standard treatment (parenteral ceftriaxone followed by oral co-amoxiclav); exploratory subgroup analysis. Figures are means (SD) unless specified otherwise

\begin{tabular}{|c|c|c|c|}
\hline Parameter & New treatment $(n=135)$ & Standard treatment $(n=143)$ & Mean difference $(95 \% \mathrm{Cl})$ \\
\hline \multicolumn{4}{|l|}{ Short term outcomes } \\
\hline Time to defervescence (hours) & $39.5(20.1)(n=134)$ & $37.2(21.8)(n=141)$ & $2.3(-2.7$ to 7.3$)$ \\
\hline White cell count $\left(\times 10^{9} / 1\right)^{*}$ & $10.0(3.7)(n=127)$ & $9.6(3.5)(n=138)$ & $0.4(-0.5$ to 1.3$)$ \\
\hline Neutrophils $\left(\times 10^{9} / \mathrm{l}\right)^{*}$ & $3.4(2.4)(n=114)$ & $3.1(2.1)(n=122)$ & $0.3(-0.3$ to 0.9$)$ \\
\hline Erythrocyte sedimentation rate (mm in first hour)* & $64.1(30.8)(n=92)$ & $59.5(27.6)(n=102)$ & $4.6(-3.7$ to 12.8$)$ \\
\hline$C$ reactive protein $(\mathrm{mg} / \mathrm{l})^{\star} \dagger$ & $10.2(11.9)(n=128)$ & $10.9(19.3)(n=141)$ & $0.7(-4.2$ to 3.1$)$ \\
\hline \multicolumn{4}{|l|}{ Primary outcome } \\
\hline Scar on renal scan at 12 months & $26 / 96(27.8 \%)$ & $33 / 100(33.0 \%)$ & $-5.8 \%(-18.7 \%$ to $6.9 \%)$ \\
\hline
\end{tabular}

*Parameters obtained 72 hours after initiation of antibiotic therapy.

tRatio between obtained value and upper limit of normal reference values for each laboratory.

\section{Secondary outcomes}

There were no significant differences between the two treatment groups for all the 502 randomised children (table 2) nor for the 278 with confirmed acute pyelonephritis on scintigraphy (table 3). We found similar results when we separately evaluated children older and younger than 2 years (data not shown). On treatment analysis showed similar results to the intention to treat analysis for primary and secondary outcomes.

Results of all available urine cultures collected after three days of treatment $(\mathrm{n}=390)$ were negative, except for two, which were both positive for Pseudomonas aeruginosa (one for each treatment group); in both cases the urine culture at onset was positive for $E$ coli and antibiotic treatment was continued as per protocol. The duration of hospital stay was similar in both groups (5.17 and 5.05 days).

\section{Adverse effects}

Fifteen children experienced minor side effects with initial treatment with co-amoxiclav (13 had diarrhoea or vomiting, or both, one had mild erythema, one had neutropenia); only 10 required a change of antibiotic. The replacement was ceftriaxone in eight patients, cefaclor in one, and cefixime in another. Three children experienced minor side effects with ceftriaxone (one had diarrhoea, one had mild erythema, and one had candida) that did not require change of treatment.

\section{Other antibiotics used}

In the new treatment group treatment was changed from co-amoxiclav to another antibiotic in a further 12 children because of antibiotic resistance (two) and intercurrent rotavirus gastroenteritis and vomiting (10). All children in the standard treatment group completed the three day course of parenteral ceftriaxone. Co-amoxiclav was not used subsequently in seven because of antibiotic resistance, in five because of intercurrent events, and in one for unknown reasons. In most cases an oral cephalosporin was used instead of co-amoxiclav.

Sixty one children received parenteral treatment for more than three days (for four days in 41 , five days in 16 , six days in two, seven days in one, and 10 days in one). In most cases this was because of the need to change occurring over a weekend or holiday period or a change in the physician in charge. It usually meant an extra dose of parenteral antibiotic the morning changeover should have occurred.

\section{DISCUSSION}

According to our definition of non-inferiority, this randomised controlled study in 502 young children with acute pyelonephritis shows that oral antibiotic treatment alone is as effective as initial parenteral treatment followed by oral antibiotics (mean risk difference for renal scarring at one year $-4 \%, 95 \%$ confidence interval $-11.1 \%$ to $3.1 \%$ ). Young children often present with symptoms of urinary tract infection, and results of initial investigations may indicate acute pyelonephritis- "inflammation of the kidney and pelvis, because of bacterial infection." 17 The prevalence of urinary tract infection among febrile infants between 2 and 24 months of age is around 5\%. ${ }^{1}$ Currently, there is no reliable and routinely applicable way of distinguishing between upper and lower tract infections at presentation, so all children should be considered as having an upper tract infection or clinical acute pyelonephritis at the start of antibiotic treatment.

Acute pyelonephritis is a serious bacterial illness because of the risk of sepsis and long term renal scarring, particularly in young children. Scarring might also be partly caused by late and inadequate treatment of episodes of acute infection. ${ }^{18}$ This has lead to extensive use of parenteral antibiotics, especially in children aged 2 or younger, for variable periods of time, up to three weeks. ${ }^{11}{ }^{19}$ In Italy parenteral antibiotics are used in around $50 \%$ of cases. ${ }^{2}$

We compared a 10 day course of oral co-amoxiclav with a three day course of parenteral treatment with ceftriaxone followed by a seven day course of oral coamoxiclav. The two treatments were equivalent in terms of our primary outcome (renal scar rate) and the short term outcomes (time to defervescence, reduction in inflammatory indices, percentage with sterile urine). These results were found in children of both sexes, aged less than 7 years. We use rate of scarring one year after the infection as the primary end point rather than the time to temperature $<37^{\circ} \mathrm{C}$ because the long term medical consequences (proteinuria, 
hypertension, chronic kidney damage) are secondary to the presence of renal scarring, which therefore represents the most important clinical end point.

We excluded extremely ill children with suspected major sepsis (such as meningococcal infection), those with documented abnormalities of the urinary tract, and those with a creatinine clearance lower than $70 \mathrm{ml} / \mathrm{min} / 1.73 \mathrm{~m}^{2}$. These exclusion criteria can be easily recognised by any physician - with the exception of the low creatinine clearance, which is an extremely rare condition - such that the results are readily applicable to children with clinical acute pyelonephritis seen in general practice. We used acute scintigraphy, the ideal method for determining pyelonephritis, to confirm upper tract infection and thus those at risk of subsequent scarring. It is not recommended as a useful test for initial decision making, however, because treatment needs to be started before scintigraphy can usually be performed.

These results were found with an intention to treat analysis, which included children who started antibiotic treatment before a scintigraphic diagnosis of acute pyelonephritis, which represents the routine clinical scenario. The doctor in charge has to decide to start antibiotic treatment in a febrile child with a positive result on urine dipstick testing and no other signs of localisation of infection because the results of urine culture are not usually available for two days. We confirmed our results in the subgroup analysis of children with the clear localisation of infection at the renal scan - that is, those children with the most severe infections.

\section{Strengths and weaknesses}

A relatively high number of children were lost at the 12 month follow-up (102/502), which is inevitable in a study of this nature. Some parents declined the followup study because of concerns regarding its invasive nature and the concomitant radiation dose. At 12 months we repeated scintigraphy only in the children at risk of scarring. Previous research has shown that children who have normal results on acute scintigraphy do not develop scarring during follow-up. ${ }^{12}$ Nevertheless, our study maintains its power in ascertaining the non-inferiority of the oral treatment, given the higher number of children enrolled and the fact that the numbers lost to follow-up were similar in both groups.

\section{Comparison with other studies}

One other study has examined the exclusive use of oral antibiotics. ${ }^{12}$ The population studied (in the United States) differed in terms of age, sex, and concomitant rate of urological abnormality. The study was restricted to children younger than 2 years, whereas our population included children up to the age of 7 , the proportion of girls was more balanced in our study $(63 \% v 90 \%)$, and we had a more typical proportion of children with vesicoureteric reflux (21\% v38\%). The US study also had a low scarring rate of $15 \%$ in children ${ }^{12}$ compared with $30 \%$ in our study, which is more usual. ${ }^{4-8}$ This finding is also inconsistent with the higher rate of vesicoureteric reflux in the US study, as vesicoureteric reflux is a recognised risk factor for renal scarring. ${ }^{1}$ The role of genetic variability in the response to infection and susceptibility to renal scarring for factors, such as the renin-angiotensin system, plasminogen activating inhibitor, TGF beta, and collagen polymorphisms, could differ between the Italian and the US population. ${ }^{18}$ Time to defervescence was considerably shorter in the US study, which is difficult to explain as we used two similar third generation cephalosporins and the clinical and laboratory (inflammatory indices) features of the two populations were similar. Also the bacterial flora responsible was similar: $E$ coli was isolated in $97.4 \%$ of cultures in the US study and in $94.4 \%$ in ours.

The recommended therapeutic approach to acute pyelonephritis is to start with parenteral antibiotics ${ }^{9-11}$ and admission to hospital. We have shown that acute pyelonephritis can be effectively treated with oral coamoxiclav, which can be easily given at home, after the collection of urine for culture. This avoids admission to hospital, resulting in reduced costs and less discomfort for the children and their family. In our study, however, all children were admitted for a mean of five days, which could have resulted in better adherence to treatment, closer clinical and laboratory assessments, and prompt treatment change as needed. Oral treatment for acute pyelonephritis at home would require close follow-up by a general practitioner.

A small proportion of children have a urinary tract infection sustained by a pathogen that is not susceptible to co-amoxiclav: in our study the resistance rate to this antibiotic amounted to $6 \%$. The risk of resistance is present in every kind of infection and the absence of a clinical response within $48-60$ hours requires reassessment of the need to admit to hospital or to change the antibiotic, according to the results of urine culture. In regions where resistance to co-amoxiclav is higher, it is appropriate to choose alternative antibiotics based on local sensitivity patterns. Few children experienced side effects of the antibiotic $(18 / 502,3.6 \%)$. The most common adverse event was gastrointestinal disturbance associated with co-amoxiclav.

\section{Conclusions}

In the management of the first diagnosed febrile urinary tract infection in children without urological abnormalities, an exclusive oral treatment is a reasonable option: the greater ease of oral treatment may facilitate care out of hospital and therefore have the potential to reduce costs and discomfort to children and their families.

We thank all the members of IRIS (Italian Renal Infection Study Group in children) who made the performance of this study possible. We particularly thank Daniela Gobber (epidemiologist) who died this year. We also thank Jennifer Hartwig, lan Hewitt, and Federica Fregonese for their help in the preparation of the manuscript, Andrea Ponzoni for statistical analysis, and Roberto Buzzetti for epidemiological advising. 


\section{WHAT IS ALREADY KNOWN ON THIS TOPIC}

Numerous studies have compared various parenteral antibiotic regimens for acute pyelonephritis in children

The only randomised controlled trial examining exclusive oral antibiotic treatment was carried out in an unusual population, with a strong female bias, and a disproportionately high incidence of vesicoureteral reflux

\section{WHAT THIS STUDY ADDS}

Treatment with oral antibiotics alone is not inferior to parenteral followed by oral treatment in the management of acute pyelonephritis in young children

Implementation of oral therapy could reduce costs and stress of admission to hospital in children
1 Downs SM. Technical report: urinary tract infections in febrile infants and young children. The Urinary Tract Subcommittee of the American Academy of Pediatrics Committee on Quality Improvement. Pediatrics 1999;103:e54.

2 Ghiro L, Cracco AT, Sartor M, Comacchio S, Zacchello G, Dall'Amico R. Retrospective study of children with acute pyelonephritis. Nephron 2002;90:8-15.

3 Wald E. Urinary tract infections in infants and children: a comprehensive overview. Curr Opin Pediatr 2004;16:85-8.

4 Stokland E, Hellstrom M, Jacobsson B, Jodal U, Sixt R. Renal damage one year after first urinary tract infection: role of dimercaptosuccinic acid scintigraphy. J Pediatr 1996;129:815-20.

5 Benador D, Neuhaus TJ, Papazyan JP, Willi UV, Engel-Bicik I, Nadal D, et al. Randomised controlled trial of 3 day versus 10 day intravenous antibiotics in acute pyelonephritis: effect on renal scarring. Arch Dis Child 2001;84:241-6.

6 Zaki M, Badawi M, Al Mutari G, Ramadan D, Rahman MA. Acute pyelonephritis and renal scarring in Kuwaiti children: a follow-up study using $99 \mathrm{mTC}$ DMSA renal scintigraphy. Pediatr Nephrol 2005;20:1116-9.

Participants of the IRIS1 study: I Marella, A Budini (Adria); L Marcazzò, S Bellato (Arzignano); G Audino, G Picco (Bassano); P Colleselli, D Scorrano (Belluno); L Pavanello (Castelfranco); C Crivellaro (Chioggia); G Cattarozzi, M Pitter, A Ballan (Dolo/Mirano); F Rossetti, V Cannella (Este/Monselice); G Svaluto-Moreolo, Caddia (Feltre); F Donzelli, F Maschio (Mestre); P Brisotto, N Crema, S Breseghella (Montebelluna); P Luxardo, A Toffolo (Oderzo); G Zacchello, G Montini, L Murer, C Carasi, B Andreetta, S Comacchio, L Rigon, S Sartori, L Tomasi, R Pertile, D Gobber (epidemiologist), A Ponzoni (statistician) (Padua); A Truini (Piove di Sacco); PG Flora, M Ranieri (San Donà); R Dall'Amico, L Donello (Thiene); G Marcer, S Zanchetta (Soave); M Del Majno, M Gheno (Venice); G Zoppi, P Fortunati (Verona-Borgo Trento); M G Santangelo, 0 Gianesini (Vicenza); A Corsini (Bentivoglio); PP Molinari (Bologna); A Zucchini (Faenza/Lugo); Venturolli (Forli); L Serra (Imola); L Casadio (Ravenna); M Principi (Macerata); M Pitschiller, W Cassar (Bolzano); M De Marini, G Crescenz (Cuneo)

Contributors: GM is guarantor; contributed to study design, recruiting the patients, data collection, analysis and interpretation of the data; and wrote the paper. AT contributed to study design, recruiting the patients, data analysis, and final approval of the article. PZ contributed to study design, data analysis, drafting and final approval of the manuscript, and interpreted DMSA scan results. RD'A conceived the study and participated in design and recruitment of patients. DG contributed to design and organisation and was involved in data analysis. AC was responsible to standardising good clinical practice for the study, provided advice on data analysis, and commented on drafts of the paper. FM, LP, PPM, DS, SZ, WC, PB, AC, SS, LDD, LM, GZ contributed to study design, data interpretation, and recruitment of patients.

Funding: Region of Veneto (research project 40/01) and association II Sogno di Stefano (Stephen's Dream)

Competing interests: None declared.

Ethical approval: Ethics committees of each participating centre approved the study protocol.
7 Rushton HG, Majd M, Jantausch B, Wiedermann BL, Belman AB. Renal scarring following reflux and nonreflux pyelonephritis in children: evaluation with $99 \mathrm{~m}$ technetium-dimercaptosuccinic acid scintigraphy. J Urol 1992;147:1327-32.

8 Jakobsson B, Berg U, Svensson L. Renal scarring after acute pyelonephritis. Arch Dis Child 1994;70:111-5.

9 Tolkoff-Rubin NE, Cotran RS, Rubin HR. In: Brenner BM, ed. The kidney. 6th ed. Philadelphia, PA: WB Saunders, 2000:1449-508.

10 Working Group of the Research Unit, Royal College of Physicians. Guidelines for the management of acute urinary tract infection in childhood. I R Coll Phys Lond 1991;25:36-42.

11 Bloomeld P, Hodson EM, Craig JC. Antibiotics for acute pyelonephritis in children. Cochrane Database Syst Rev 2005;(1):CD003772.

12 Hoberman A, Wald ER, Hickey RW, Baskin M, Charron M, Majd M, et al. Oral versus initial intravenous therapy for urinary tract infections in young febrile children. Pediatrics 1999;104:79-86.

13 Winberg J, Anderson HJ, Bergstrom T, Jacobsson B, Larson H, Lincoln K. Epidemiology of symptomatic urinary tract infection in childhood. Acta Paediatr Scand Suppl 1974;63(252):1-20.

14 Nathan DG, Hoskin SH. Pediatric hematology. 5th ed. Philadelphia PA: WB Saunders, 1998, appendix 28.

15 Schwartz GJ, Haycock GB, Edelmann CM Jr, Spitzer A. A simple estimate of glomerular filtration rate in children derived from body length and plasma creatinine. Pediatrics 1976;58:259.

16 D'Agostino RB, Massaro JM, Sullivan LM. Non-inferiority trials: design concepts and issues - the encounters of academic consultants in statistics. Statist Med 2003;22:169-86.

17 Dorlands. Illustrated medical dictionary. 30th ed. Philadelphia PA: WB Saunders, 2003.

18 Jahnukainen T, Chen M, Celsi G. Mechanisms of renal damage owing to infection. Pediatr Nephrol 2005;20:1043-53.

19 Craig JC, Hodson EM. Treatment of acute pyelonephritis in children. BM/ 2004;328:179-80.

Accepted: 17 May 2007 